\title{
FORMAL WEIERSTRASS NON-INTEGRABILITY CRITERION FOR SOME CLASSES OF POLYNOMIAL DIFFERENTIAL SYSTEMS IN $\mathbb{C}^{2}$
}

\author{
JAUME GINÉ ${ }^{1}$ AND JAUME LLIBRE ${ }^{2}$
}

\begin{abstract}
In this paper we present a criterion for determining the formal Weierstrass non-integrability of some polynomial differential systems in the plane $\mathbb{C}^{2}$. The criterion uses solutions of the form $y=f(x)$ of the differential system in the plane and their associated cofactors, where $f(x)$ is a formal power series. In particular the criterion provides necessary conditions in order that some polynomial differential systems in $\mathbb{C}^{2}$ be formal Weierstrass integrable. Inside this class there exists non-Liouville integrable systems. Finally we extend the theory of formal Weierstrass integrability to formal Puiseux Weierstrass integrability.
\end{abstract}

\section{INTRODUCTION}

To determine when a differential system in $\mathbb{C}^{2}$ has or has not a first integral is one of the main problems in the qualitative theory of differential systems. The Liouville integrability is one of the most important theories of integrability starting with Darboux in [8,9] and strongly developed during the last decades $[10,15,29]$. This theory is based on the existence of invariant algebraic curves and their multiplicity through the exponential factors. Recently generalizations on the Liouville integrability theory have been done in several works, see [17, 18, 24, 25, 30].

Of course there exist differential systems which are integrable, i.e. with an explicit first integral, and that are non-Liouville integrable, an example is given below. Hence a natural question is: How to detect these non-Liouville integrable systems? In this work we shall see that this detection is possible for some class of polynomial differential

2010 Mathematics Subject Classification. Primary 34C05. Secondary 37C10.

Key words and phrases. Weierstrass integrability, Liouville integrability, polynomial differential systems. 
systems through a new criterion that detects formal Weierstrass nonintegrability. We also apply the criterion to some polynomial differential systems. But we must start providing some preliminary definitions and results.

Consider the polynomial differential system in the plane $\mathbb{C}^{2}$

$$
\dot{x}=P(x, y), \quad \dot{y}=Q(x, y),
$$

where the functions $P$ and $Q$ are polynomials in the complex variables $x$ and $y$, i.e. $P, Q \in \mathbb{C}[x, y]$. The degree of system (1) is $m=\max \{\operatorname{deg} P, \operatorname{deg} Q\}$. Obviously system (1) has the associated differential equation

$$
\frac{\mathrm{d} y}{\mathrm{~d} x}=\frac{Q(x, y)}{P(x, y)},
$$

and the associated vector field $\mathcal{X}=P(x, y) \partial / \partial x+Q(x, y) \partial / \partial y$.

We say that $f(x, y)=0$ with $f \in \mathbb{C}[x, y]$, is an invariant algebraic curve of system (1), or equivalently of equation (2), if the orbital derivative $\dot{f}=\mathcal{X} f=P \partial f / \partial x+Q \partial f / \partial y$ vanishes on $f=0$. In particular the algebraic curve $f(x, y)=0$ is invariant if by the Hilbert's Nullstellensatz [3] there exists a polynomial $K(x, y) \in \mathbb{C}[x, y]$ of degree less than or equal to $m-1$, called the cofactor associated to the curve $f(x, y)=0$ such that

$$
\mathcal{X} f=P \frac{\partial f}{\partial x}+Q \frac{\partial f}{\partial y}=K f
$$

A non-locally constant function $H: U \subset \mathbb{C}^{2} \rightarrow \mathbb{C}$ is a first integral of system (1) in the open set $U$ if this function is constant in each solution $(x(t), y(t))$ of system $(1)$ contained in $U$. Clearly $H \in C^{1}(U)$ is a first integral of system (1) on $U$ if and only if $\mathcal{X} H=P \partial H / \partial x+Q \partial H / \partial y \equiv 0$ on $U$.

A function $R$ is an integrating factor associated to a first integral $H$ of system (1) if

$$
R P=-\frac{\partial H}{\partial y}, \quad \text { and } \quad R Q=\frac{\partial H}{\partial x},
$$

or equivalently

$$
P \frac{\partial R}{\partial x}+Q \frac{\partial R}{\partial y}=-\left(\frac{\partial P}{\partial x}+\frac{\partial Q}{\partial y}\right) R=-\operatorname{div}(\mathcal{X}) R
$$


We recall that a polynomial differential system (1) has a Liouvillian first integral $H$ if its associated integrating factor is of the form

$$
R=\exp \left(\frac{D}{E}\right) \prod_{i} C_{i}^{\alpha_{i}},
$$

where $D, E$ and the $C_{i}$ are polynomials in $\mathbb{C}[x, y]$ and $\alpha_{i} \in \mathbb{C}$, see for more details $[5,16,27,29]$. The functions of the form (5) are called Darboux functions. Note that the curves $C_{i}=0$ are invariant algebraic curves of the polynomial differential system (1), and the exponential $\exp (D / E)$ is a product of some exponential factors associated to the invariant algebraic curves of system (1) or to the invariant straight line at infinity when such invariant curves have multiplicity greater than one, for more details see $[4,6,7]$ or Chapter 8 of [10].

However there are differential systems without a Liouvillian first integral, see for instance $[11,20,26]$. A simple example is the polynomial Liénard differential system

$$
\dot{x}=-y+x^{4}, \quad \dot{y}=x,
$$

that has no integrating factors of the form (5), and consequently is not Liouvillian integrable, see [11] for more details.

These type of examples are included in the first generalization of the Liouvillian theory of integrability where the cofactors for non-algebraic invariant curves are defined, see [11]. The problem in this generalization is how to detect these non-algebraic invariant curves. Later on the Liouvillian theory of integrability has been extended to $n$-dimensional autonomous or non-autonomous differential systems in [18]. A method for detecting non-algebraic invariant curves for some polynomial differential systems was given in [12].

The next question is whether a non-algebraic invariant curve has always a polynomial cofactor. The answer to this question in general is negative, see an example given in [18].

Let $\mathbb{C}[[x]]$ be the set of the formal power series in the variable $x$ with coefficients in $\mathbb{C}$, and $\mathbb{C}[y]$ the set of the polynomials in the variable $y$ with coefficients in $\mathbb{C}$. A function of the form

$$
\sum_{i=0}^{\ell} a_{i}(x) y^{i} \in \mathbb{C}[[x]][y]
$$

is called a formal Weierstrass polynomial in $y$ of degree $\ell$. Here we have privileged the variable $y$ but of course we can privileged the variable $x$ instead of $y$. A formal Weierstrass polynomial whose coefficients 
are convergent is called a Weierstrass polynomial, for more details see $[2,17,18]$.

The expression of a cofactor of an invariant curve $y-g(x)=0$ where $g(x)$ is a formal Weierstrass polynomial is given in the following result proved in [13].

Proposition 1. Let $g(x) \in \mathbb{C}[[x]]$. An invariant curve of the form $y-g(x)=0$ of a polynomial differential system (1) of degree $m$ has a formal Weierstrass polynomial cofactor of the form

$$
K(x, y)=k_{m-1}(x) y^{m-1}+\cdots+k_{1}(x) y+k_{0}(x) .
$$

In [17] it is given a generalization of the Liouvillian integrability based in the following definition. A planar autonomous differential system is (formal) Weierstrass integrable if admits an integrating factor of the form (5) where $D, E$ and the $C_{i}$ 's are (formal) Weierstrass polynomials.

Note that there are systems which are Weierstrass integrable and are not Liouvillian integrable, see for instance system (6) and example 2 below. In the papers [19, 20, 21, 23] are studied some Liénard differential systems and Abel differential equations that are Weierstrass integrable.

Let $\mathbb{C}[[x, y]]$ be the set of all formal power series in the variables $x$ and $y$ with coefficients in $\mathbb{C}$. The following result is given in [2].

Theorem 2. If $f \in \mathbb{C}[[x, y]]$ then it has a unique decomposition of the form

$$
f=u x^{r} \prod_{j=1}^{\ell}\left(y-g_{j}(x)\right),
$$

where $g_{j}(x)$ are Puiseux series and $r \in \mathbb{Z}, r \geq 0$ and $u \in \mathbb{C}[[x, y]]$ is invertible inside the ring $\mathbb{C}[[x, y]]$.

We note that a Darboux integrating factor (5) is analytic function where it is defined consequently by Theorem 2 it can be written into the form (9).

We say that a polynomial differential system (1) is weakly formal Weierstrass integrable if it has an integrating factor of the form

$$
R=\prod_{i=1}^{\ell}\left(y-f_{i}(x)\right)^{\alpha_{i}},
$$


where the functions $f_{i}(x) \in \mathbb{C}[[x]]$.

In this work we give a criterion for detecting when a polynomial differential system (1) is not weakly formal Weierstrass integrable. We note that in general to detect the non-integrability of a differential system is a very difficult problem.

\section{THE CRITERION}

The criterion is based in the following result which is an extension of the results of Darboux integrability for formal invariant curves, see for instance [10].

Theorem 3. Assume that the polynomial differential system (1) is weakly formal Weierstrass integrable, that is, it has an integrating factor of the form (10). If $K_{i}$ is the cofactor of any formal invariant curve $y-f_{i}(x)=0$, then

$$
\sum_{i=1}^{\ell} \alpha_{i} K_{i}=-\operatorname{div}(\mathcal{X})
$$

Proof. The existence of the integrating factor $R$, given in (10), forces that the curve $y-f_{i}(x)=0$, for $i=1, \ldots, \ell$, is invariant for system (1), and consequently by Proposition 4 it has a formal Weierstrass polynomial cofactor $K_{i}$ such that

$$
\mathcal{X}\left(y-f_{i}(x)\right)=K_{i}\left(y-f_{i}(x)\right) .
$$

Since $R$ is an integrating factor, from (4), we have

$$
\begin{aligned}
-\operatorname{div}(\mathcal{X}) R & =\mathcal{X}(R)=\mathcal{X}\left(\prod_{i=1}^{\ell}\left(y-f_{i}(x)\right)^{\alpha_{i}}\right) \\
& =\sum_{i=1}^{\ell} \mathcal{X}\left(\left(y-f_{j}(x)\right)^{\alpha_{j}}\right) \prod_{i=1, i \neq j}^{\ell}\left(y-f_{i}(x)\right)^{\alpha_{i}} \\
& =\sum_{i=1}^{\ell} \alpha_{i}\left(y-f_{j}(x)\right)^{\alpha_{j}-1} \mathcal{X}\left(y-f_{j}(x)\right) \prod_{i=1, i \neq j}^{\ell}\left(y-f_{i}(x)\right)^{\alpha_{i}} \\
& =\sum_{i=1}^{\ell} \alpha_{i}\left(y-f_{j}(x)\right)^{\alpha_{j}-1} K_{i}\left(y-f_{j}(x)\right) \prod_{i=1, i \neq j}^{\ell}\left(y-f_{i}(x)\right)^{\alpha_{i}} \\
& =\sum_{i=1}^{\ell} \alpha_{i} K_{i} \prod_{i=1}^{\ell}\left(y-f_{i}(x)\right)^{\alpha_{i}}=\left(\sum_{i=1}^{\ell} \alpha_{i} K_{i}\right) R .
\end{aligned}
$$


This completes of the proof of the theorem.

Our criterion for detecting polynomial differential systems which are not weakly formal Weierstrass integrable works as follows. First we compute the solutions of the form $y=f_{i}(x)=g_{i}(x)+\mathcal{O}\left(x^{r+1}\right)=$ $\sum_{j=0}^{r} a_{j} x^{j}+\mathcal{O}\left(x^{r+1}\right) \in \mathbb{C}[[x]]$ of the polynomial differential system (1) through the equation $E q:=\dot{x} d y / d x-\dot{y}=0$ up to order $r$ in the variable $x$. After we compute the corresponding cofactor $L_{i} \in \mathbb{C}[[x]][y]$ of the invariant curve $y-f_{i}(x)=0$ up to order $r$, using the equality

$$
\mathcal{X}\left(y-g_{i}(x)\right)=L_{i}\left(y-g_{i}(x)\right)+\mathcal{O}\left(x^{r+1}\right) .
$$

From Theorem 3 if the cofactors $L_{i}$ 's do not verify the equality

$$
\sum_{i=1}^{\ell} \alpha_{i} L_{i}=-\operatorname{div}_{r}(\mathcal{X})+\mathcal{O}\left(x^{r+1}\right),
$$

where $\operatorname{div}_{r}(\mathcal{X})$ is the divergence $\operatorname{div}(\mathcal{X})$ up to order $r$ in the variables $x$ and $y$, then system (1) cannot be weakly formal Weierstrass integrable with $f_{i}(x)$ formal series.

\section{Puiseux Weierstrass integrability}

In this section we propose a generalization of the formal Weierstrass integrability that contains all the Liouville integrability.

Let $\mathbb{C}((x))$ be the set of series in fractionary powers in the variable $x$ with coefficients in $\mathbb{C}$ (these series are called Puiseux series), and $\mathbb{C}[y]$ the set of the polynomials in the variable $y$ with coefficients in $\mathbb{C}$. We call a function of the form

$$
\sum_{i=0}^{\ell} a_{i}(x) y^{i} \in \mathbb{C}((x))[y]
$$

a Puiseux Weierstrass polynomial in $y$ of degree $\ell$. Here we have privileged the variable $y$ but of course we can privileged the variable $x$ instead of $y$. This definition is a generalization of the formal Weierstrass polynomial introduced before.

The expression of a cofactor of an invariant curve $y-g(x)=0$ where $g(x)$ is a Puiseux Weierstrass polynomial is given in the following result which a generalization of Proposition 1.

Proposition 4. Let $g(x) \in \mathbb{C}((x))$. An invariant curve of the form $y-g(x)=0$ of a polynomial differential system (1) of degree $m$ has a 
Puiseux Weierstrass polynomial cofactor of the form

$$
K(x, y)=k_{m-1}(x) y^{m-1}+\cdots+k_{1}(x) y+k_{0}(x) .
$$

Proof. The proof of this proposition is exactly the same than Proposition 1.4 of [13].

A planar autonomous differential system is Puiseux Weierstrass integrable if it admits an integrating factor of the form (5) where $D, E$ and the $C_{i}$ 's are Puiseux Weierstrass polynomials. By definition the Puiseux Weierstrass integrable systems include the Liouville integrable systems.

The criterion based in Theorem 3 is also generalizable for such integrable systems. In a future work we will compute the invariant solutions $y-f_{i}(x)$ of a polynomial differential system (1) when the functions $f_{i}(x) \in \mathbb{C}((x))$. The hard problem in this case is in what fractionary power begins the Puiseux series $f_{i}(x)$.

\section{EXAMPLES}

Example 1. Now consider the polynomial differential system

$$
\dot{x}=-y+x^{2}, \quad \dot{y}=x+a x^{2} .
$$

This system has a focus at the origin because the first Poincaré-Liapunov constant is $V_{4}=a$, see for more details on the Liapunov constants the Chapter 4 of [10]. Now we study if system (14) can be weakly formal Weierstrass integrable, that is, with $f_{i}(x) \in \mathbb{C}[[x]]$. Following the criterion we propose a solution curve of the form

$$
y=f(x)=a_{0}+a_{1} x+a_{2} x^{2}+a_{3} x^{3}+a_{4} x^{4}+a_{5} x^{5}+\cdots
$$

up to a fixed order $r$ in the variable $x$. Substituting this solution in the first ordinary differential equation $E q:=\dot{x} d y / d x-\dot{y}=0$ we get an infinite system of equations. In order to determine the coefficients $a_{i}$ up to certain order we fix the developments of $f(x)$ and $E q$. If we do that up to order 5 and we solve the finite system of equations, we find the following four solutions curves for system (14):

$$
\begin{aligned}
& \text { 1) } y=y_{1}(x)=\frac{3\left(-5+\sqrt{1-8 a^{2}}\right)}{\left(8\left(3+a^{2}\right)\right.}+\frac{1}{6}\left(5+\sqrt{1-8 a^{2}}\right) x^{2}+\frac{1}{9} a(5+ \\
& \left.\sqrt{1-8 a^{2}}\right) x^{3}+\frac{1}{27}\left(2\left(-1+\sqrt{1-8 a^{2}}\right)-a^{2}\left(9+\sqrt{1-8 a^{2}}\right)\right) x^{4}- \\
& \frac{4}{405} a\left(-29-25 \sqrt{1-8 a^{2}}+a^{2}\left(57+5 \sqrt{1-8 a^{2}}\right)\right) x^{5}+\mathcal{O}\left(x^{6}\right) \text {, }
\end{aligned}
$$


2) $y=y_{2}(x)=\frac{3\left(-5-\sqrt{1-8 a^{2}}\right)}{\left(8\left(3+a^{2}\right)\right.}+\frac{1}{6}\left(5-\sqrt{1-8 a^{2}}\right) x^{2}+\frac{1}{9} a(5-$ $\left.\sqrt{1-8 a^{2}}\right) x^{3}+\frac{1}{27}\left(2\left(-1-\sqrt{1-8 a^{2}}\right)-a^{2}\left(9+\sqrt{1-8 a^{2}}\right)\right) x^{4}-$ $\frac{4}{405} a\left(29-25 \sqrt{1-8 a^{2}}+a^{2}\left(-57+5 \sqrt{1-8 a^{2}}\right)\right) x^{5}+\mathcal{O}\left(x^{6}\right)$,

3) $y=y_{3}(x)=-i x-i(i+a) x^{2} / 3+i\left(2-i a+a^{2}\right) x^{3} / 18-i(-8 i+6 a-$ $\left.\left.9 i a^{2}+5 a^{3}\right) x^{4} / 270+i\left(-12-76 i a+3 a^{2}-66 i a^{3}+25 a^{4}\right) x^{5}\right) / 3240+$ $\mathcal{O}\left(x^{6}\right)$,

4) $y=y_{4}(x)=i x+i(-i+a) x^{2} / 3-i\left(2+i a+a^{2}\right) x^{3} / 18+i(8 i+6 a+$ $\left.\left.9 i a^{2}+5 a^{3}\right) x^{4} / 270-i\left(-12+76 i a+3 a^{2}+66 i a^{3}+25 a^{4}\right) x^{5}\right) / 3240+$ $\mathcal{O}\left(x^{6}\right)$.

Next we compute their Weierstrass polynomial cofactors up to order 5 and we find that

1) $K_{1}=\frac{1}{81}\left(x\left(135+135 a x-24 x^{2}-108 a^{2} x^{2}+116 a x^{3}-228 a^{3} x^{3}\right)+\right.$ $\left.\sqrt{1-8 a^{2}} x\left(-27-27 a x-24 x^{2}+12 a^{2} x^{2}-100 a x^{3}+20 a^{3} x^{3}\right)\right)+$ $\mathcal{O}\left(x^{6}\right)$

2) $K_{2}=\frac{1}{81}\left(x\left(135+135 a x-24 x^{2}-108 a^{2} x^{2}+116 a x^{3}-228 a^{3} x^{3}\right)-\right.$ $\left.\sqrt{1-8 a^{2}} x\left(-27-27 a x-24 x^{2}+12 a^{2} x^{2}-100 a x^{3}+20 a^{3} x^{3}\right)\right)+$ $\mathcal{O}\left(x^{6}\right)$

3) $K_{3}=\frac{1}{3240}\left(2\left(1080 x+270 a x^{2}-192 x^{3}-216 a^{2} x^{3}+190 a x^{4}+\right.\right.$ $\left.165 a^{3} x^{4}\right)+i\left(3240+2160 a x-1080 x^{2}-540 a^{2} x^{2}+288 a x^{3}+\right.$ $\left.\left.240 a^{3} x^{3}+60 x^{4}-15 a^{2} x^{4}-125 a^{4} x^{4}\right)\right)+\mathcal{O}\left(x^{6}\right)$,

4) $K_{4}=\frac{1}{3240}\left(2\left(1080 x+270 a x^{2}-192 x^{3}-216 a^{2} x^{3}+190 a x^{4}+\right.\right.$ $\left.165 a^{3} x^{4}\right)+i\left(-3240-2160 a x+1080 x^{2}+540 a^{2} x^{2}-288 a x^{3}-\right.$ $\left.\left.240 a^{3} x^{3}-60 x^{4}+15 a^{2} x^{4}+125 a^{4} x^{4}\right)\right)+\mathcal{O}\left(x^{6}\right)$.

Now we try to see if there is a linear combination of them equal to minus the divergence, that is

$$
c_{1} K_{1}+c_{2} K_{2}+c_{3} K_{3}+c_{4} K_{4}=-\operatorname{div}_{5} \mathcal{X}+\mathcal{O}\left(x^{6}\right),
$$

and this equation does not have any solution. Hence by the criterion system (14) has not an integrating factor of the form (10). Consequently system (14) is not weakly formal Weierstrass integrable. 
When $a=0$ equation (16) has the solution

$$
c_{1}=c_{2}=-\frac{5}{2}\left(1+c_{4}\right) \quad c_{3}=1+c_{4} .
$$

In fact for $a=0$ the differential system (14) is a time-reversible system because is invariant by the the symmetry $(x, y, t) \rightarrow(-x, y,-t)$. Hence it has a center at the origin. Moreover it has a Darboux integrating factor of the form $R=\left(1+2 y-2 x^{2}\right)^{-1}$. So for $a=0$ system (14) is Liouvillian integrable.

Example 2. Consider the differential system (6). This system is time-reversible because it is invariant under the symmetry $(x, y, t) \rightarrow$ $(-x, y,-t)$. Hence it has a center at the origin of coordinates. Moreover it has not a Liouvillian first integral, see [11].

Now we are going to apply the criterion to detect if system (6) has an integrating factor of the form $(10)$ with $f_{i}(x) \in \mathbb{C}[[x]]$.

We propose a solution curve of the form $y=\sum_{j=0}^{4} a_{j} x^{j}+\mathcal{O}\left(x^{5}\right)$ and substituting this solution into the differential equation $E q:=\dot{x} d y / d x-$ $\dot{y}=0$ we get an infinite system of equations. If we stop this system taking into account the equations up to order 4 , and solve this finite system of equations we obtain the following five solutions curves:

1) $y=y_{1}(x)=-i x+x^{4} / 5+\mathcal{O}\left(x^{5}\right)$,

2) $y=y_{2}(x)=i x+x^{4} / 5+\mathcal{O}\left(x^{5}\right)$,

3) $y=y_{3}(x)=1 / 2(-3)^{1 / 3}+(-1)^{2 / 3} x^{2} / 3^{1 / 3}+x^{4} / 3+\mathcal{O}\left(x^{5}\right)$,

4) $y=y_{4}(x)=-3^{1 / 3} / 2+x^{2} / 3^{1 / 3}+x^{4} / 3+\mathcal{O}\left(x^{5}\right)$,

5) $y=y_{5}(x)=-1 / 2(-1)^{2 / 3} 3^{1 / 3}-(-1)^{1 / 3} x^{2} / 3^{1 / 3}+x^{4} / 3+\mathcal{O}\left(x^{5}\right)$.

Now we are going to compute the Weierstrass polynomial cofactors of these solution curves up to order 4 . Since system (6) is of degree 4 the Weierstrass polynomial cofactors are of the form $K=k_{0}(x)+k_{1}(x) y+$ $k_{2}(x) y^{2}+k_{3}(x) y^{3}$. Applying equation (3) to the invariant solutions $y-y_{k}(x)=\mathcal{O}\left(x^{5}\right)$ for $k=1, \ldots, 5$, we obtain for each of these solutions curves their Weierstrass polynomial cofactor up to order 4, i.e.

1) $K_{1}=\left(-5 i+4 x^{3}\right) / 5+\mathcal{O}\left(x^{5}\right)$,

2) $K_{2}=\left(5 i+4 x^{3}\right) / 5+\mathcal{O}\left(x^{5}\right)$,

3) $K_{3}=\left(3^{7 / 6} i x-3^{2 / 3} x+4 x^{3}\right) / 3+\mathcal{O}\left(x^{5}\right)$,

4) $K_{4}=2\left(3^{2 / 3} x+2 x^{3}\right) / 3+\mathcal{O}\left(x^{5}\right)$.

5) $K_{5}=\left(-3^{7 / 6} i x-3^{2 / 3} x+4 x^{3}\right) / 3+\mathcal{O}\left(x^{5}\right)$, 
Now we study if system (6) satisfies equation (11). Solving the equation

$$
c_{1} K_{1}+c_{2} K_{2}+c_{3} K_{3}+c_{4} K_{4}+c_{5} K_{5}=-\operatorname{div}_{4} \mathcal{X}+\mathcal{O}\left(x^{5}\right),
$$

we get the solution $c_{3}=c_{4}$ and

$$
\begin{aligned}
& c_{1}=-\frac{15 i-5 \sqrt{3}+6 i c_{4}-2 \sqrt{3} c_{4}+10 i c_{5}}{5(-i+\sqrt{3})}, \\
& c_{2}=\frac{10 \sqrt{3}+4 \sqrt{3} c_{4}+5 i c_{5}+5 \sqrt{3} c_{5}}{5 i-5 \sqrt{3}} .
\end{aligned}
$$

Consequently system (6) could have an integrating factor of the form (10) with $f_{i}(x) \in \mathbb{C}[[x]]$. We claim that this system is Weierstrass integrable because it has the integrating factor

$$
R(x, y)=f_{2}^{-2}(x, y)
$$

Indeed, if $\operatorname{Ai}(z)$ and $\operatorname{Bi}(z)$ are the two independent Airy functions, see a definition in [1], then we know that system system (6) is integrable because it has the first integral $H(x, y)=f_{1} f_{2}^{-1}$, where

$$
\begin{aligned}
& f_{1}(x, y)=-(-2)^{1 / 3} x^{2} \operatorname{Ai}\left((-2)^{2 / 3} y\right)+\operatorname{Ai}^{\prime}\left((-2)^{2 / 3} y\right) \\
& f_{2}(x, y)=(-2)^{1 / 3} x^{2} \operatorname{Bi}\left((-2)^{2 / 3} y\right)-\operatorname{Bi}^{\prime}\left((-2)^{2 / 3} y\right) .
\end{aligned}
$$

The non-algebraic curves $f_{i}(x, y)=0$ are invariant curves by the flow of system (6), satisfying the corresponding equation (3) for the vector field $\mathcal{X}$ associated to system (6) with cofactors $K_{1}=K_{2}=2 x^{3}$. Consequently it has the integrating factor given in (17), which is which is not of the form (5). In short the polynomial differential system (6) is formal Weierstrass integrable but not Liouvillian integrable.

\section{ACKNowledGements}

The first author is partially supported by a MINECO/ FEDER grant number MTM2017-84383-P and an AGAUR (Generalitat de Catalunya) grant number 2017SGR-1276. The second author is supported by the Ministerio de Economía, Industria y Competitividad, Agencia Estatal de Investigación grants MTM2016-77278-P (FEDER) and MDM-2014-0445, the Agència de Gestió d'Ajuts Universitaris i de Recerca grant 2017SGR1617, and the H2020 European Research Council grant MSCA-RISE-2017-777911. 


\section{REFERENCES}

[1] M. Abramowitz and I. A. Stegun, Handbook of mathematical functions with formulas, graphs, and mathematical tables, National Bureau of Standards Applied Mathematics Series 55, U.S. Government Printing Office, Washington, D.C. 1964

[2] E. Casas-Alvero, Singularities of Plane Curves, London Mathematical Society Lecture Note Series 276, Cambridge University Press, 2000.

[3] M. Atiyah and I.G. Macdonald, Introduction to Commutative Algebra, Addison- Wesley, 1994.

[4] J. Chavarriga, H. Giacomini, J. Giné, Darboux integrability and the inverse integrating factor, J. Differential Equations 194 (2003), no. 1, 116-139.

[5] C.J. Christopher, Liouvillian first integrals of second order polynomial differential equations, Electron. J. Differential Equations 1999, No. 49, 7 pp.

[6] C.J. Christopher, J. Llibre, Algebraic aspects of integrability for polynomial systems, Qual. Theory Dyn. Syst. 1 (1999), no. 1, 71-95.

[7] C.J. Christopher, J. Llibre, J.V. Pereira, Multiplicity of invariant algebraic curves in polynomial vector fields, Pacific J. of Math. 229 (2007), no. $1,63-117$.

[8] G. Darboux, Mémoire sur les équations différentielles algébrique du premier ordre et du premier degré (Mélanges), Bull. Sci. Math. 2ème série, 2 (1878), 60-96; 123-144; 151-200.

[9] G. Darboux, De l'emploi des solutions particulières algébriques dans l'intégration des systèmes d'équations différentielles algébriques, C. R. Math. Acad. Sci. Paris 86 (1878), 1012-1014.

[10] F. Dumortier, J. Llibre, J.C. Artés, Qualitative theory of planar differential systems, UniversiText, Springer-Verlag, New York, 2006.

[11] I.A. García, J. Giné, Generalized cofactors and nonlinear superposition principles, Appl. Math. Lett. 16 (2003), no. 7, 1137-1141.

[12] I.A. García, J. GinÉ, Non-algebraic invariant curves for polynomial planar vector fields, Discrete Contin. Dyn. Syst. 10 (2004), no. 3, 755-768.

[13] I.A. García, H. Giacomini, J. Giné, Generalized nonlinear superposition principles for polynomial planar vector fields, J. Lie Theory 15 (2005), no. 1, 89-104.

[14] H. Giacomini, J. Giné, M. Grau, The role of algebraic solutions in planar polynomial differential systems, Math. Proc. Cambridge Philos. Soc. 143 (2007), no. 2, 487-508.

[15] J. GiNé, On some open problems in planar differential systems and Hilbert's 16th problem, Chaos Solitons Fractals 31 (2007), no. 5, 1118-1134.

[16] J. Giné, Reduction of integrable planar polynomial differential systems, Appl. Math. Lett. 25 (2012), no. 11, 1862-1865.

[17] J. Giné, M. Grau, Weierstrass integrability of differential equations, Appl. Math. Lett. 23 (2010), no. 5, 523-526.

[18] J. Giné, M. Grau, J. Llibre, On the extensions of the Darboux theory of integrability, Nonlinearity 26 (2013), no. 8, 2221-2229.

[19] J. Giné, J. Llibre, Weierstrass integrability in Liénard differential systems, J. Math. Anal. Appl. 377 (2011), no. 1, 362-369.

[20] J. Giné, J. LliBRE, On the mechanisms for producing linear type centers in polynomial differential systems, Mosc. Math. J. 18 (2018), no. 3, 1-12. 
[21] J. Giné, X. Santallusia, Abel differential equations admitting a certain first integral, J. Math. Anal. Appl. 370 (2010), no. 1, 187-199.

[22] J. Giné, X. Santallusia, Essential variables in the integrability problem of planar vector fields, Phys. Lett. A 375 (2011), no. 3, 291-297.

[23] J. Llibre, C. VAlls, Generalized Weierstrass integrability of the Abel differential equations, Mediterr. J. Math. 10 (2013), no. 4, 1749-1760.

[24] J. Llibre, X. Zhang, Darboux theory of integrabiltity in $\mathbb{C}^{n}$ taking into account the multiplicity, J. Differential Equations 246 (2009), 541-551.

[25] J. Llibre, X. Zhang, Rational first integrals in the Darboux theory of integrability in $\mathbb{C}^{n}$, Bull. Sci. Math. 134 (2010), 189-195.

[26] G.R. Nicklason, An Abel type cubic system Electron. J. Differential Equations 2015, No. 189, 17 pp.

[27] M.J. Prelle, M.F. Singer, Elementary first integrals of differential equations, Trans. Amer. Math. Soc. 279 (1983), 613-636.

[28] A. Seidenberg, Reduction of singularities of the differential equation $A d y=$ Bdx, Amer. J. Math. 90 (1968), 248-269.

[29] M.F. Singer, Liouvillian first integrals of differential equations, Trans. Amer. Math. Soc. 333 (1992), 673-688.

[30] X. ZHANG, Integrability of dynamical systems: algebra and analysis, in Developments in Mathematics 47, Sringer, 2017.

1 Departament de Matemàtica, Inspires Research Centre, Universitat de Lleida, Avda. Jaume II, 69; 25001 Lleida, Catalonia, Spain

Email address: gine@matematica.udl.cat

2 Departament de Matemàtiques, Universitat Autònoma de Barcelona, 08193 Bellaterra, Barcelona, Catalonia, Spain

Email address: jllibre@mat.uab.cat 